\title{
Low dietary energy density is associated with high dietary quality in Irish adults
}

\author{
L. Keyes, J. Walton and A. Flynn \\ School of Food and Nutritional Sciences, University College Cork, Cork, Republic of Ireland
}

Low dietary energy density (DED) has been associated with higher dietary quality. The objective of this analysis was to examine the dietary quality of Irish adults with low, medium or high DED using data from the National Adult Nutrition Survey (NANS) (http:// www.iuna.net).

Dietary intake data were analysed using WISPC (Tinuviel Software, Anglesey, UK) that contains McCance and Widdowson's The Composition of Foods, 6th edition ${ }^{(1)}$. DED $(\mathrm{kJ} / \mathrm{g})$ was calculated using food only, excluding all beverages. Participants were stratified by gender and age and separated by tertile of DED into categories of low, medium and high DED. The mean daily intakes (MDI) of food, macronutrients, dietary fibre and micronutrients were examined across tertile of DED.

MDI of energy, macronutrient, dietary fibre and wt. of food consumed, by tertile of DED in Irish adults

\begin{tabular}{|c|c|c|c|c|c|c|c|c|}
\hline \multirow[b]{2}{*}{ Mean DED (kJ/g) } & \multicolumn{2}{|c|}{ Low $(n=499) 5.48$} & \multicolumn{2}{|c|}{ Medium $(n=502) 7.07$} & \multicolumn{2}{|c|}{ High $(n=499) 8.83$} & \multirow[b]{2}{*}{ p-value* } & \\
\hline & Mean & S.D. & Mean & S.D. & Mean & S.D. & & \\
\hline Wt. food ex. beverages (g) & $1262^{\mathrm{a}}$ & 411 & $1038^{b}$ & 300 & $887^{c}$ & 276 & 0.000 & $\downarrow$ \\
\hline Energy (kcal) & $1864^{\mathrm{a}}$ & 608 & $2028^{b}$ & 644 & $2150^{c}$ & 686 & 0.000 & $\uparrow$ \\
\hline Protein (\%TE) & $18.5^{\mathrm{a}}$ & 4.2 & $17.2^{\mathrm{b}}$ & 3.3 & $15.5^{\mathrm{c}}$ & 3.0 & 0.000 & $\downarrow$ \\
\hline Fat (\%TE) & $32.1^{\mathrm{a}}$ & 5.9 & $34.4^{\mathrm{b}}$ & 5.7 & $37.5^{\mathrm{c}}$ & 5.9 & 0.000 & $\uparrow$ \\
\hline Saturated fat (\%TE) & $11.6^{\mathrm{a}}$ & 3.2 & $12.8^{b}$ & 3.1 & $14.4^{\mathrm{c}}$ & 3.4 & 0.000 & $\uparrow$ \\
\hline Carbohydrate (\%TE) & $44.0^{\mathrm{a}}$ & 7.0 & $42.9^{b}$ & 6.7 & $41.7^{\mathrm{c}}$ & 6.8 & 0.000 & $\downarrow$ \\
\hline Total sugar (\%TE) & $18.1^{\mathrm{a}}$ & 5.8 & $16.4^{b}$ & 5.4 & $16.0^{b}$ & 5.9 & 0.000 & $\downarrow$ \\
\hline Dietary fibre (g/10 MJ) & $27.7^{\mathrm{a}}$ & 8.7 & $23.0^{b}$ & 6.7 & $19.2^{\mathrm{c}}$ & 6.0 & 0.000 & $\downarrow$ \\
\hline Vitamin A $(\mu \mathrm{g} / 10 \mathrm{MJ})$ & $1940^{\mathrm{a}}$ & 2067 & $1294^{b}$ & 945 & $1023^{\mathrm{c}}$ & 1046 & 0.000 & $\downarrow$ \\
\hline Vitamin D (mg/10 MJ) & $8.6^{\mathrm{a}}$ & 13.9 & $5.0^{\mathrm{b}}$ & 5.1 & $4.1^{\mathrm{c}}$ & 4.9 & 0.000 & $\downarrow$ \\
\hline Vitamin E (mg/10 MJ) & $22.8^{\mathrm{a}}$ & 58.5 & $12.2^{b}$ & 13.0 & $10.4^{\mathrm{c}}$ & 7.4 & 0.000 & $\downarrow$ \\
\hline Thiamin $(\mathrm{mg} / 10 \mathrm{MJ})$ & $6.2^{\mathrm{a}}$ & 17.3 & $2.8^{b}$ & 5.3 & $2.2^{\mathrm{c}}$ & 3.5 & 0.000 & $\downarrow$ \\
\hline Riboflavin (mg/10 MJ) & $6.1^{\mathrm{a}}$ & 16.4 & $3.0^{b}$ & 5.2 & $2.4^{\mathrm{c}}$ & 3.0 & 0.000 & $\downarrow$ \\
\hline Niacin Equivalents (mg/10 MJ) & $65.9^{a}$ & 53.1 & $52.4^{\mathrm{b}}$ & 14.2 & $46.4^{\mathrm{c}}$ & 14.5 & 0.000 & $\downarrow$ \\
\hline Vitamin B6 (mg/10 MJ) & $7.9^{\mathrm{a}}$ & 20.9 & $4.0^{b}$ & 5.4 & $3.4^{\mathrm{c}}$ & 4.5 & 0.000 & $\downarrow$ \\
\hline Vitamin B12 (mg/10 MJ) & $13.9^{\mathrm{a}}$ & 59.8 & $7.3^{b}$ & 6.5 & $6.3^{\mathrm{c}}$ & 4.4 & 0.000 & $\downarrow$ \\
\hline Folate $(\mu \mathrm{g} / 10 \mathrm{MJ})$ & $567^{\mathrm{a}}$ & 616 & $475^{\mathrm{b}}$ & 1036 & $362^{\mathrm{c}}$ & 176 & 0.000 & $\downarrow$ \\
\hline Vitamin C (mg/10 MJ) & $259.7^{\mathrm{a}}$ & 473.7 & $135.3^{b}$ & 197.6 & $75.7^{\mathrm{c}}$ & 72.6 & 0.000 & $\downarrow$ \\
\hline Calcium (mg/10 MJ) & $1266^{\mathrm{a}}$ & 569 & $1113^{b}$ & 349 & $1046^{\mathrm{c}}$ & 353 & 0.000 & $\downarrow$ \\
\hline Magnesium (mg/10 MJ) & $410^{a}$ & 126 & $342^{b}$ & 65 & $301^{\mathrm{c}}$ & 66 & 0.000 & $\downarrow$ \\
\hline $\operatorname{Iron}(\mathrm{mg} / 10 \mathrm{MJ})$ & $22.0^{\mathrm{a}}$ & 30.0 & $16.9^{b}$ & 18.1 & $14.6^{\mathrm{c}}$ & 13.9 & 0.000 & $\downarrow$ \\
\hline Sodium $(\mathrm{mg} / 10 \mathrm{MJ})$ & 3150 & 889 & 3171 & 708 & 3172 & 700 & 0.214 & \\
\hline Potassium (mg/10 MJ) & $4232^{\mathrm{a}}$ & 983 & $3655^{b}$ & 596 & $3209^{c}$ & 614 & 0.000 & $\downarrow$ \\
\hline Zinc $(\mathrm{mg} / 10 \mathrm{MJ})$ & $15.7^{\mathrm{a}}$ & 14.9 & $12.0^{\mathrm{b}}$ & 4.3 & $10.4^{\mathrm{c}}$ & 3.3 & 0.000 & $\downarrow$ \\
\hline
\end{tabular}

Low DED was associated with higher intakes of protein, carbohydrates, dietary fibre, vitamins and minerals and lower intakes of fat and saturated fat. This analysis shows that a low energy dense diet is associated with higher dietary quality.

The project was funded by the Irish Government under the Food for Health Research Initiative 2007-2012.

1. Food Standards Agency (2002) McCance and Widdowson's The Composition of Foods 6th ed. Cambridge: Royal Society of Chemistry. 